\title{
Comunicación
}

\section{Parámetros hematológicos de referencia de cuyes nativos (Cavia porcellus)}

\author{
Hematological reference parameters of indigenous guinea pigs (Cavia porcellus) \\ Katherine Patricia Oriundo Núñez ${ }^{1,3}$, Pedro Mauricio Delgadillo Coronado ${ }^{1}$, \\ Renzo André Arévalo Pérez ${ }^{1}$, Miriam Ibet Alfaro-Astorima ${ }^{1,2}$, \\ Sandra Bautista Gómez ${ }^{1}$
}

\section{Resumien}

El presente estudio tuvo como objetivo determinar los parámetros hematológicos de cuyes nativos de la zona de Ayacucho, Perú, teniendo en cuenta la edad, sexo y estado fisiológico (preñez, recría, destetado). Se tomaron muestras de sangre y se analizaron manualmente siguiendo los métodos rutinarios de laboratorio. El recuento de glóbulos rojos y los valores de hematocrito y hemoglobina fueron mayores en cuyes de la etapa recría en comparación con cuyes destetados, y en machos en comparación con hembras. Asimismo, el recuento de glóbulos rojos y los valores de hematocrito y hemoglobina de hembras preñadas fueron similares a los valores de cuyes de recría. En la fórmula leucocitaria, los linfocitos fueron los de mayor proporción, seguidos de los neutrófilos. Los valores de referencia reportados en los diferentes grupos etarios y estado fisiológico, permitirá la toma de mejores decisiones clínicas.

Palabras clave: hemoglobina, hematocrito, recuento de glóbulos rojos, linfocitos

\footnotetext{
${ }^{1}$ Instituto Nacional de Innovación Agraria, Estación Experimental Agraria Canaán, Ayacucho, Perú

${ }^{2}$ Escuela Profesional de Medicina Veterinaria, Universidad Nacional de San Cristóbal de Huamanga, Ayacucho, Perú

${ }^{3}$ E-mail: catypat@hotmail.com
}

Recibido: 4 de agosto de 2020

Aceptado para publicación: 4 de septiembre de 2021

Publicado: 27 de octubre de 2021

CLos autores. Este artículo es publicado por la Rev Inv Vet Perú de la Facultad de Medicina Veterinaria, Universidad Nacional Mayor de San Marcos. Este es un artículo de acceso abierto, distribuido bajo los términos de la licencia Creative Commons Atribución 4.0 Internacional (CC BY 4.0) [https:// creativecommons.org/licenses/by/4.0/deed.es] que permite el uso, distribución y reproducción en cualquier medio, siempre que la obra original sea debidamente citada de su fuente original 
The present study aimed to determine the haematological parameters of indigenous guinea pigs in the Ayacucho area, Peru, taking into account age, sex and physiological status (pregnancy, lactating, weaned). Blood samples were taken and analysed manually following routine laboratory methods. The red blood cell count and haematocrit and haemoglobin values were higher in guinea pigs of the lactating stage compared to weaned animals, and in males as compared to females. Likewise, the red blood cell count and the haematocrit and haemoglobin values of pregnant females were like those of lactating guinea pigs. In the leukocyte formula, lymphocytes had the highest frequency, followed by neutrophils. The reference values reported in the different age groups and physiological status will allow better clinical decisions to be made.

Key words: haemoglobin, haematocrit, red blood cell count, lymphocytes

\section{INTRODUCCIÓN}

La identificación de patologías que afectan la productividad en animales de producción, en la mayoría de los casos, se realiza mediante la historia y la observación de signos clínicos, lo cual en algunas ocasiones genera diagnósticos errados. Los exámenes de laboratorio, entre ellos los hematológicos, contribuyen a un mejor diagnóstico de enfermedades infecciosas y parasitarias, constituyendo un «primer paso» diagnóstico, tanto en medicina humana como animal (Medway et al., 1986; Williams et al., 2016).

Los estudios de componentes sanguíneos, además de mostrar el estado de salud del paciente, pueden usarse como herramientas de monitoreo de los cambios fisiológicos que ocurren en los animales (Bezerra et al., 2008). Factores fisiológicos como la gestación, lactancia, edad y sexo han sido mencionados en distintas especies animales como causantes de variaciones en los valores hematológicos normales (Novozhilov et al., 2012; Guzmán y Callacná, 2013; He et al., 2017; Herrera et al., 2017; Argente et al., 2019). La evaluación de los parámetros hematológicos permite conocer la capacidad de las especies y grupos raciales para adaptarse a diferentes entornos (Delfino et al., 2012; Ayvar, 2018).

La producción de cuyes no es ajena a la presentación de patologías que afectan la productividad, generando pérdidas económicas en los productores. Para poder realizar un diagnóstico rápido y seguro, es necesario contar con información referencial de los valores hematológicos. En este sentido, Genzer et al. (2019) indican que la edad y el sexo del animal afectan los valores de glóbulos rojos, hemoglobina y hematocrito; Tapia (2019) y Vidalón (2014) sugieren considerar la altitud y situación geográfica para obtener valores de referencia reales para cada zona, en tanto que Ayvar (2018) indica que la suplementación de probióticos en la dieta incide en los parámetros hematológicos $\mathrm{y}$ bioquímicos nutricionales, siendo todos estos trabajos realizados en cuyes.

Los cuyes nativos presentan índices productivos y reproductivos inferiores con relación a las razas mejoradas (Mendoza, 2012), pero por otro lado, tienen ciertas ventajas (resistencia a enfermedades, rusticidad, versatilidad para el consumo de diversos tipos de 
alimentos), debido al proceso de adaptación a los diferentes pisos ecológicos (INIA, 2009).

Existe poca información sobre los parámetros hematológicos de cuyes de acuerdo con la edad y estado fisiológico en condiciones de altura, pues la mayoría de las publicaciones están relacionadas con otros pisos altitudinales. En tal sentido, el objetivo del presente estudio fue determinar los valores hematológicos en condiciones de altura de cuyes nativos (Cavia porcellus) por edad, sexo y estado fisiológico.

\section{Materiales y Métodos}

\section{Lugar de Estudio y Animales}

El estudio se realizó en la provincia de Huamanga, departamento de Ayacucho, Perú, zona que se encuentra a una altitud de $2735 \mathrm{msnm}$. Se emplearon cuyes nativos del banco de germoplasma de la Estación Experimental Agraria Canaán, Ayacucho. Los cuyes se encontraban sometidos a un sistema de crianza intensiva, alojados en pozas de $1.50 \mathrm{~m}$ de largo x $1 \mathrm{~m}$ de ancho y $0.50 \mathrm{~m}$ de alto. La alimentación fue a base de alfalfa suministrada ad libitum y el agua era ofrecida en pocillos de arcilla.

Los cuyes se encontraban en aparente buen estado de salud. Se seleccionaron 20 cuyes de recría de tres meses de edad aproximadamente (10 machos - 10 hembras), 20 cuyes destetados de aproximadamente 1 mes de edad (10 machos - 10 hembras) y nueve cuyes preñadas

\section{Muestras de Sangre}

La obtención de las muestras se realizó a primeras horas del día, previo a la alimentación de los animales. Las muestras fueron obtenidas mediante punción de la vena cefálica en tubos vacutainer con anticoagulante EDTA y agujas $\mathrm{N}^{\circ}$. 21. Se extrajo 1 $\mathrm{ml}$ de sangre por cuy. Las muestras fueron llevadas al laboratorio en un cooler con gel refrigerante.

En el laboratorio se procedió a realizar los siguientes análisis:

- Hematocrito: Se realizó el llenado de tubos capilares con heparina, los que fueron centrifugados a $10000 \mathrm{~g}$ durante 10 minutos. La lectura se hizo con ayuda de la tabla de microhematocrito, cuya escala varía de 0 a 100 .

- Hemoglobina: Se dividió el valor del hematocrito por el factor 3 (Forrellat et al., 2010) para obtener la concentración de hemoglobina.

- Recuento total de glóbulos rojos: Se realizó una dilución de 1:200 con el reactivo Hayem en tubos de ensayo (1990 $\mu 1$ del reactivo y $10 \mu 1$ de sangre), se homogenizó la muestra y se incubó durante 5 minutos. La muestra obtenida fue colocada en la cámara de Neubauer para realizar la lectura de los cuadrantes de la cámara bajo el microscopio. El resultado obtenido fue multiplicado por 10000 (Washington y Hoosier, 2012).

- Recuento total de glóbulos blancos: Se realizó una dilución de 1:20 con el reactivo de Turk en tubos de ensayo ( 380 $\mu 1$ del reactivo y $20 \mu 1$ de sangre), se homogenizó la muestra y se incubó durante 5 minutos. La muestra obtenida fue colocada en la cámara de Neubauer para su lectura. El resultado obtenido fue multiplicado por 50 .

- Recuento diferencial de glóbulos blancos: Se realizó el frotis sanguíneo en una lámina portaobjeto. El frotis se dejó secar a temperatura ambiente. Se realizó la tinción con Wright, dejándolo en reposo durante 5 minutos. Se realizó el conteo de 100 células y el resultado fue expresado en porcentaje.

- Índices eritrocitarios: Los índices eritrocitarios volumen corpuscular medio (VCM), hemoglobina corpuscular media (HCM) y concentración de hemoglobina corpuscular medio (CHCM) fueron calculados utilizando las fórmulas descritas por Washington y Hoosier (2012). 
Cuadro 1. Parámetros hematológicos de cuyes nativos de la etapa recría (Huamanga, Ayacucho, Perú)

\begin{tabular}{|c|c|c|c|c|c|c|c|c|}
\hline & \multicolumn{4}{|c|}{ Machos } & \multicolumn{4}{|c|}{ Hembras } \\
\hline & Media & DS & Mín. & Máx. & Media & DS & Mín. & Máx. \\
\hline $\begin{array}{l}\text { Glóbulos rojos } \\
\left(\mathrm{x} 10^{6} / \mu \mathrm{l}\right)\end{array}$ & $5.63^{\mathrm{a}}$ & 0.34 & 4.83 & 6.02 & $4.36^{\mathrm{b}}$ & 0.32 & 3.60 & 4.70 \\
\hline Hematocrito (\%) & $46.70^{\mathrm{a}}$ & 1.73 & 44.00 & 49.00 & $39.70^{\mathrm{b}}$ & 2.65 & 34.00 & 42.00 \\
\hline $\begin{array}{l}\text { Hemoglobina } \\
(\mathrm{g} / \mathrm{dl})\end{array}$ & $14.15^{\mathrm{a}}$ & 0.53 & 13.33 & 14.85 & $13.06^{\mathrm{b}}$ & 0.87 & 11.20 & 13.80 \\
\hline VCM (fl) & $83.25^{\mathrm{b}}$ & 6.64 & 74.45 & 99.38 & $91.11^{\mathrm{a}}$ & 1.53 & 89.36 & 94.40 \\
\hline HCM (pg) & $30.31^{\mathrm{a}}$ & 0.01 & 30.30 & 30.31 & $29.98^{\mathrm{a}}$ & 0.53 & 29.36 & 31.10 \\
\hline CHCM (g/dl) & $25.23^{\mathrm{b}}$ & 2.01 & 22.55 & 30.12 & $32.90^{\mathrm{a}}$ & 0.06 & 32.78 & 33.00 \\
\hline $\begin{array}{l}\text { Glóbulos blancos } \\
\left(\mathrm{x} 10^{3} / \mu \mathrm{l}\right)\end{array}$ & $6.02^{b}$ & 1.29 & 3.95 & 8.25 & $7.45^{\mathrm{a}}$ & 0.36 & 6.83 & 7.97 \\
\hline $\begin{array}{l}\text { Segmentados } \\
(\%)\end{array}$ & $11.90^{\mathrm{a}}$ & 8.94 & 1.00 & 33.00 & $9.80^{\mathrm{a}}$ & 3.06 & 6.00 & 15.00 \\
\hline $\begin{array}{l}\text { Abastonados } \\
(\%)\end{array}$ & 0 & & & & & & & \\
\hline Linfocitos (\%) & $82.70^{\mathrm{a}}$ & 10.81 & 57.00 & 94.00 & $84.90^{\mathrm{a}}$ & 4.25 & 78.00 & 91.00 \\
\hline Monocitos (\%) & $4.00^{\mathrm{a}}$ & 3.03 & 1.00 & 10.00 & $4.40^{\mathrm{a}}$ & 1.74 & 2.00 & 7.00 \\
\hline Eosinófilos (\%) & 1.40 & 0.49 & 1.00 & 2.00 & 0.80 & 0.60 & 0.00 & 2.00 \\
\hline Basófilos (\%) & 0 & 0 & 0 & 0 & 0 & 0 & 0 & 0 \\
\hline
\end{tabular}

DS: Desviación estándar; VCM: Volumen corpuscular medio; HCM: Hemoglobina corpuscular media; CHCM: Concentración de hemoglobina corpuscular medio

a,b Superíndices diferentes indican que diferencias estadísticas (prueba de Tukey, $P<0.05$ )

\section{Análisis Estadístico}

Se utilizaron los promedios como medidas de tendencias central y las desviaciones estándar como medidas de dispersión para el análisis de los datos encontrados. Para evaluar las diferencias en los valores hematológicos por sexo y grupo etario se realizó el análisis de varianza y la comparación de medias mediante la prueba Tukey $(\mathrm{p}<0.05)$.

\section{Resultados y Discusión}

Los parámetros hematológicos de cuyes nativos de la etapa de recría (machos y hem bras) se presentan en el Cuadro 1. Ayvar (2018) reportó un recuento de glóbulos rojos de $5.75 \pm 0.31 \times 10^{6} / \mu 1$ y un hematocrito de $46.67 \pm 3.0 \%$ en cuyes machos, resultados similares a los del presente estudio. Asimismo, Williams et al. (2016) reportaron valores de $48.7 \pm 0.33$ de hematocrito y recuento de glóbulos rojos de $5.69 \pm 0.039 \times 10^{6} / \mu 1$, resultados cercanos a los del presente estudio.

Vidalón (2014) obtuvo valores del recuento de glóbulos rojos en cuyes machos (2.5 meses) de $6.08 \pm 0.32 \times 10^{6} / \mu 1$ para la línea cárnica y de $6.09 \pm 0.38 \times 10^{6} / \mu 1$ para la línea precoz, y un hematocrito de $56.7 \pm 2.64 \%$ ) para la línea cárnica y de $53.5 \pm 2.98 \%$ para 
Cuadro 2. Parámetros hematológicos de cuyes nativos destetados (Huamanga, Ayacucho, Perú)

\begin{tabular}{|c|c|c|c|c|c|c|c|c|}
\hline & \multicolumn{4}{|c|}{ Machos } & \multicolumn{4}{|c|}{ Hembras } \\
\hline & Media & DS & Mín. & Máx. & Media & DS & Mín. & Máx. \\
\hline $\begin{array}{l}\text { Glóbulos rojos } \\
\left(\times 10^{6} / \mu \mathrm{l}\right)\end{array}$ & $3.94^{\mathrm{a}}$ & 0.60 & 2.80 & 4.70 & $3.81^{\mathrm{a}}$ & 0.64 & 2.60 & 4.60 \\
\hline Hematocrito (\%) & $35.70^{\mathrm{a}}$ & 5.42 & 25.00 & 43.00 & $35.00^{\mathrm{a}}$ & 5.81 & 24.00 & 42.00 \\
\hline $\begin{array}{l}\text { Hemoglobina } \\
(\mathrm{g} / \mathrm{dl})\end{array}$ & $11.72^{\mathrm{a}}$ & 1.80 & 8.10 & 14.10 & $11.49^{\mathrm{a}}$ & 1.95 & 7.80 & 13.80 \\
\hline VCM (fl) & $90.63^{\mathrm{a}}$ & 1.34 & 89.20 & 93.90 & $91.93^{\mathrm{a}}$ & 2.45 & 88.24 & 96.67 \\
\hline HCM (pg) & $29.75^{\mathrm{a}}$ & 0.52 & 28.93 & 30.91 & $30.17^{\mathrm{a}}$ & 0.84 & 28.80 & 31.70 \\
\hline CHCM (g/dl) & $32.82^{\mathrm{a}}$ & 0.16 & 32.40 & 32.97 & $32.82^{\mathrm{a}}$ & 0.14 & 32.50 & 33.00 \\
\hline $\begin{array}{l}\text { Glóbulos blancos } \\
\left(\mathrm{x} 10^{3} / \mu \mathrm{l}\right)\end{array}$ & $6.50^{\mathrm{b}}$ & 0.45 & 5.93 & 7.36 & $7.05^{\mathrm{a}}$ & 0.51 & 5.89 & 7.67 \\
\hline $\begin{array}{l}\text { Segmentados } \\
(\%)\end{array}$ & $11.20^{\mathrm{a}}$ & 4.75 & 6.00 & 20.00 & $10.80^{\mathrm{a}}$ & 2.36 & 8.00 & 15.00 \\
\hline $\begin{array}{l}\text { Abastonados } \\
(\%)\end{array}$ & 0 & 0 & 0 & 0 & 0 & 0 & 0 & 0 \\
\hline Linfocitos $(\%)$ & $86.00^{\mathrm{a}}$ & 4.75 & 78.00 & 92.00 & $86.50^{\mathrm{a}}$ & 2.69 & 82.00 & 92.00 \\
\hline Monocitos (\%) & 2.80 & 1.25 & 1.00 & 5.00 & 2.80 & 2.18 & 0 & 8.00 \\
\hline Eosinófilos (\%) & 0.10 & 0.30 & 0 & 1.00 & 0.10 & 0.30 & 0 & 1.00 \\
\hline Basófilos (\%) & 0 & 0 & 0 & 0 & 0.10 & 0.30 & 0 & 1.00 \\
\hline
\end{tabular}

DS: Desviación estándar; VCM: Volumen corpuscular medio; HCM: Hemoglobina corpuscular media; $\mathrm{CHCM}$ : Concentración de hemoglobina corpuscular medio

a,b Superíndices diferentes indican que diferencias estadísticas (prueba de Tukey, $\mathrm{P}<0.05$ )

la línea precoz, valores superiores a los de este estudio, posiblemente debido a que dicho trabajo se realizó a una altitud de 3350 msnm, lo que generó un aumento considerable de producción de eritrocitos para captar mayor cantidad de oxígeno. En este sentido, Tapia (2019) y Matute (2019) indican que una mayor altitud produce una eritrocitosis absoluta secundaria ante una mayor demanda de oxígeno.

En la etapa de recría se observó un mayor recuento de glóbulos rojos, y valores de hematocrito y hemoglobina en machos en comparación a las hembras $(\mathrm{p}<0.05$; Cuadro
1), más no así en los cuyes destetados (Cuadro 2). Según Genzer et al. (2019), los cuyes machos alcanzaron mayores valores de glóbulos rojos, hematocrito, hemoglobina, recuento de glóbulos blancos y monocitos. Asimismo, Kitagaki et al. (2005) reportaron un mayor recuento de glóbulos rojos y blancos en machos que en hembras.

Ayvar (2018) reportó $10.65 \pm 4.71 \times 10^{3} / \mu 1$ de glóbulos blancos, mientras que Vidalón (2014) obtuvo un recuento de $9.6 \pm 2.53 \times 10^{3} / \mu 1$, valores que fueron superiores a los encontrados en el presente estudio. Personett et al. (2019) también reportaron recuentos to- 
Cuadro 3. Parámetros hematológicos de cuyes machos de la etapa de recría y destete (Huamanga, Ayacucho, Perú)

\begin{tabular}{|c|c|c|c|c|}
\hline & \multicolumn{2}{|c|}{ Recría } & \multicolumn{2}{|c|}{ Destetados } \\
\hline & Media & DS & Media & $\mathrm{DS}$ \\
\hline Glóbulos rojos $\left(\times 10^{6} / \mathrm{mm}^{3}\right)$ & $5.63^{a}$ & 0.34 & $3.94^{\mathrm{b}}$ & 0.60 \\
\hline Hematocrito (\%) & $46.70^{\mathrm{a}}$ & 1.73 & $35.70^{b}$ & 5.42 \\
\hline Hemoglobina (g/dl) & $14.15^{\mathrm{a}}$ & 0.53 & $11.72^{b}$ & 1.80 \\
\hline VCM (fl) & $83.25^{b}$ & 6.64 & $90.63^{\text {a }}$ & 1.34 \\
\hline $\mathrm{HCM}(\mathrm{pg})$ & $30.31^{\mathrm{a}}$ & 0.01 & $29.75^{b}$ & 0.52 \\
\hline $\mathrm{CHCM}(\mathrm{g} / \mathrm{dl})$ & $25.23^{\mathrm{b}}$ & 2.01 & $32.82^{\mathrm{a}}$ & 0.16 \\
\hline Glóbulos blancos $\left(\mathrm{x} 10^{3} / \mathrm{mm}^{3}\right)$ & $6.02^{\mathrm{a}}$ & 1.29 & $6.50^{\mathrm{a}}$ & 0.45 \\
\hline Segmentados $(\%)$ & $11.90^{\mathrm{a}}$ & 8.94 & $11.20^{\mathrm{a}}$ & 4.75 \\
\hline Abastonados (\%) & 0.00 & 0.00 & 0.00 & 0.00 \\
\hline Linfocitos (\%) & $82.70^{\mathrm{a}}$ & 10.81 & $86.00^{\mathrm{a}}$ & 4.75 \\
\hline Monocitos (\%) & $4.00^{\mathrm{a}}$ & 3.03 & $2.80^{\mathrm{a}}$ & 1.25 \\
\hline Eosinófilos (\%) & 1.40 & 0.49 & 0.10 & 0.30 \\
\hline Basófilos (\%) & 0.00 & 0.00 & 0.00 & 0.00 \\
\hline
\end{tabular}

DS: Desviación estándar; VCM: Volumen corpuscular medio; HCM: Hemoglobina corpuscular media; CHCM: Concentración de hemoglobina corpuscular medio

a,b Superíndices diferentes indican que diferencias estadísticas (prueba de Tukey, $\mathrm{P}<0.05$ )

tales de glóbulos blancos superiores $\left(12.79 \pm 0.74 \times 10^{3} / \mu \mathrm{l}\right)$, posiblemente debido al uso de $5 \%$ de isoflurano para inmovilizar a los animales y obtener la muestra. Hoyos et al. (2001), al evaluar diversas concentraciones de isoflurano, evidenció incremento en el recuento de glóbulos blancos, además otros cambios en la serie roja. De otra parte, Williams et al. (2016) reportaron valores de $5.89 \pm 0.17 \times 10^{3} / \mu 1$ glóbulos blancos, los cuales son similares a los del presente estudio.

Se obtuvo un valor de $14.15 \pm 0.53 \mathrm{~g} / \mathrm{dl}$ de hemoglobina en cuyes machos de recría. En este sentido, Ayvar (2018) reportó $15.59 \pm 0.88 \mathrm{~g} / \mathrm{dl}$ de hemoglobina, Vidalón (2014) obtuvo $16.4 \pm 0.81$ y $15.8 \pm 0.81 \mathrm{~g} / \mathrm{dl}$ para cuyes de la líneas cárnica y precoz, respectivamente., en todos los casos resultados superiores a los del presente estudio, posiblemente debido a que los cuyes en dichos estu- dios se encontraban en mejor plano de alimentación (Medway et al., 1986). Asimismo, Personett et al. (2019) reportan $15.46 \pm 0.55 \mathrm{~g} / \mathrm{dl}$ de hemoglobina en cuyes machos y hembras de bioterio.

En cuanto al sexo, los machos mostraron mayores niveles de hematocrito, hemoglobina y volumen corpuscular medio (VCM), resultado similar al estudio de Spittler et al. (2021), quienes estudiaron las diferencias asociadas con la edad y el sexo en los parámetros hematológicos de cuyes Dunkin Hartley; sin embargo, esta diferencia solo se observó en la etapa de recría.

Se observaron diferencias relacionadas con la edad (Cuadros 3 y 4 ) donde el recuento de glóbulos rojos, hematocrito y hemoglobina se incrementan acorde al desarrollo del animal. Estos resultados están de acuerdo con 
Cuadro 4. Parámetros hematológicos de cuyes hembras de la etapa de recría, destete y preñada (Huamanga, Ayacucho, Perú)

\begin{tabular}{|c|c|c|c|c|c|c|}
\hline & \multicolumn{2}{|c|}{ Preñada } & \multicolumn{2}{|c|}{ Recría } & \multicolumn{2}{|c|}{ Destetados } \\
\hline & Media & DS & Media & DS & Media & DS \\
\hline Glóbulos rojos $\left(\mathrm{x} 10^{6} / \mu \mathrm{l}\right)$ & $4.63^{\mathrm{a}}$ & 0.30 & $4.36^{\mathrm{a}}$ & 0.32 & $3.81^{\mathrm{b}}$ & 0.64 \\
\hline Hematocrito (\%) & $42.33^{\mathrm{a}}$ & 2.26 & $39.70^{\mathrm{a}}$ & 2.65 & $35.00^{\mathrm{b}}$ & 5.81 \\
\hline Hemoglobina (g/dl) & $13.93^{\mathrm{a}}$ & 0.76 & $13.06^{\mathrm{a}}$ & 0.87 & $11.49^{b}$ & 1.95 \\
\hline VCM (fl) & $90.94^{\mathrm{a}}$ & 1.10 & $91.11^{\mathrm{a}}$ & 1.53 & $91.93^{\mathrm{a}}$ & 2.45 \\
\hline $\mathrm{HCM}(\mathrm{pg})$ & $29.93^{\mathrm{a}}$ & 0.37 & $29.98^{\mathrm{a}}$ & 0.53 & $30.17^{\mathrm{a}}$ & 0.84 \\
\hline $\mathrm{CHCM}(\mathrm{g} / \mathrm{dl})$ & $32.90^{\mathrm{a}}$ & 0.05 & $32.90^{\mathrm{a}}$ & 0.06 & $32.82^{\mathrm{a}}$ & 0.14 \\
\hline Glóbulos blancos $\left(\mathrm{x} 10^{3} / \mu \mathrm{l}\right)$ & $7.54^{\mathrm{a}}$ & 0.57 & $7.45^{\mathrm{a}}$ & 0.36 & $7.05^{\mathrm{a}}$ & 0.51 \\
\hline Segmentados (\%) & $8.67^{\mathrm{a}}$ & 2.05 & $9.80^{\mathrm{a}}$ & 3.06 & $10.80^{\mathrm{a}}$ & 2.36 \\
\hline Abastonados (\%) & 0.00 & 0.00 & 0.00 & 0.00 & 0.00 & 0.00 \\
\hline Linfocitos (\%) & $87.11^{\mathrm{a}}$ & 3.14 & $84.90^{\mathrm{a}}$ & 4.25 & $86.50^{\mathrm{a}}$ & 2.69 \\
\hline Monocitos (\%) & 4.00 & 1.25 & 4.40 & 1.74 & 2.80 & 2.18 \\
\hline Eosinófilos (\%) & 0.56 & 0.68 & 0.80 & 0.60 & 0.10 & 0.30 \\
\hline Basófilos (\%) & 0.11 & 0.31 & 0.00 & 0.00 & 0.10 & 0.30 \\
\hline
\end{tabular}

DS: Desviación estándar; VCM: Volumen corpuscular medio; HCM: Hemoglobina corpuscular media; CHCM: Concentración de hemoglobina corpuscular medio

a,b Superíndices diferentes indican que diferencias estadísticas (prueba de Tukey, $\mathrm{P}<0.05$ )

los obtenidos por otros autores; así, Zimmerman et al. (2019) reporta recuento de glóbulos rojos de 4.36-6.84 × 10\%/ $\mu$ en machos adultos, 3.35-6.15 x 10\%/ $\mu$ l en hembras adultas y $4.06-6.02 \times 10^{6} / \mu 1$ en cuyes de $2-90$ días y de hematocrito de 37-47, 40.9-49.9 y $33.8-48.8 \%$ para adultos machos y hembras y menores de 90 días, respectivamente. Genzer et al. (2019) indican, asimismo, que el recuento de glóbulos rojos, hemoglobina, hematocrito y el volumen medio de plaquetas aumenta con la edad. Asimismo, Spittler et al. (2021) señala que en comparación con animales jóvenes, los adultos tenían valores de mayores de hematocrito, recuento de glóbulos rojos y concentración de hemoglobina.

Los resultados en hembras, según edad y estado reproductivo, muestran que los valores de glóbulos rojos, hematocrito, hemoglobina fueron similares a aquellos de cuyes en etapa de recría y mayores a los observados en cuyes en etapa de destete $(p<0.05$; Cuadro 4). Couto (2010) reporta que el estado fisiológico altera los niveles de leucocitos, encontrando leucocitosis en animales preñados. Verastegui (2015), de otra parte, reportó un recuento de glóbulos rojos de 5.3 x $10^{6} / \mu 1$ y de glóbulos blancos de $9.9 \times 10^{3} / \mu 1$, así como valores de hematocrito de $40.6 \% \mathrm{y}$ de hemoglobina de $13.6 \mathrm{~g} / \mathrm{dl}$ en cuyes hembras gestantes.

En el recuento diferencial de glóbulos blancos, se encontró que las células con mayor proporción fueron los linfocitos (Cuadros 1-4), seguido de los neutrófilos, tal y como ha sido reportado por Zimmerman et al. (2015). Kitagaki et al. (2005) reportan 55.3 $\pm 10.1 \%$ de linfocitos, $35.5 \pm 10.0 \%$ de neutrófilos, $2.8 \pm 2.2 \%$ de monocitos, $5.4 \pm 2.6 \%$ de eosinófilos y $0.6 \pm 0.8 \%$ de basófilos en cuyes 
machos; Vidalón (2014) obtuvo un recuento de linfocitos de $54.8 \pm 16.9 \%$ para la Línea Precoz y de $59.7 \pm 11.2 \%$ para la Línea Cárnica; Genzer et al. (2019) reporta en cuyes hembras $49 \pm 11,45.5 \pm 10.5,3.5 \pm 1.2$, $2.2 \pm 1.1$ y $0.2 \pm 0.2 \%$ de linfocitos, neutrófilos, monocitos, eosinófilos y basófilos respectivamente y en machos de $50 \pm 9,43 \pm 9,4.5 \pm 1.7$, $1.0 \pm 0.6$ y $0.2 \pm 0.2 \%$ de linfocitos, neutrófilos, monocitos, eosinófilos y basófilos, respectivamente. Asimismo, Zimmerman et al. (2019) reporta valores de referencia en cuyes de 290 días de linfocitos (52.6-83.2), neutrófilos (14.8-42.6\%), monocitos (0-3.7\%), eosinófilos (0.1-3.6\%) y basófilos $(0-0.58 \%)$.

Finalmente, Takisawa et al. (2004), quienes establecieron valores medios de recuentos sanguíneos en cuyes, remarcan que en general, el recuento de glóbulos blancos y el porcentaje de linfocitos son más bajos que en otros roedores, pero que el porcentaje de granulocitos es más alto.

\section{Conclusiones}

- Existen diferencias en los valores hematológicos en cuyes de la etapa recría, entre machos y hembras.

- Se debe tener en cuenta el factor sexo y edad en el análisis de los parámetros hematológicos, lo que permitirá realizar un mejor diagnóstico.

\section{Literatura Citada}

1. Argente MJ, Abad DM, Bermejo E, García ML, López A. 2019. Reference values for selected blood parameters in rabbits: effects of age and physiological status. Ind J Anim Res B-1165. doi: 10.18805/ijar.B-1165

2. Ayvar JE. 2018. Parámetros hematológicos y bioquímicos nutricionales en $\mathrm{Ca}$ via porcellus suplementados con probiótico Lactobacillus spp. Tesis de Médico Veterinario. Lima: Univ. Ricar- do Palma. 72 p.

3. Bezerra LR, Ferreira AF, Camboim EK, Justiniano SV, Machado PC, Gomes BB. 2008. Perfil hematológico de cabras clinicamente sadias criadas no Cariri paraibano. Cienc Agrotec 32: 955-960.

4. Couto A. 2010. Caracterización genética y perfil hematológico y bioquímico en ovinos de raza «Criolla lanada serrana» del Planalto serrano Catarinense - Santa Catarina, Brasil. Tesis Doctoral. León, España: Univ. de León. 375 p.

5. Delfino L, De Souza B, Da R, Silva $W$. 2012. Efeito do estresse calórico sobre o eritrograma de ruminantes. Agropecuária Científica no Semiárido 8: 1-7.

6. Forrellat-Barrios M, HernándezRamírez P, Fernández-Delgado N, Pita-Rodríguez G 2010. ¿Se cumple siempre la relación hemoglobina hematocrito? Rev Cuban Hematol Inmunol Hemoter 26: 359-361.

7. Guzmán LE, Callacná MA. 2013. Valores hematológicos de cabras criollas en dos estados fisiológicos reproductivos. Sci Agropec 4: 285-292.

8. Genzer S, Huynh T, Coleman J, Harmon J, Welch S, Spengler J. 2019. Hematology and clinical chemistry reference intervals for inbred strain 13/ $\mathrm{N}$ guinea pigs (Cavia porcellus). J Am Assoc Lab Anim 58: 293-303. doi: 10.30802/AALAS-JAALAS-18-000118

9. He Q, Su G, Liu K, Zhang F, Jiang Y, Gao J, Liu L, et al. 2017. Sex-specific reference intervals of hematologic and biochemical analytes in Sprague-Dawley rats using the nonparametric rank percentile method. PLoS One 12: e0189837. doi: 10.1371/journal.pone.0189837

10. Herrera Y, Rugeles C, Vergara O. 2017. Perfil hematológico del burro crio1lo (Equus asinus) colombiano. Rev Colomb Cienc Anim 9: 158-163. doi: 10.24188/recia.v9.n2.2017.553

11. Hoyos M, Brumbaugh G, Meza G, Sumano H. 2001. Inducción experimental de anestesia general en potros con la administración intravenosa de 
isoflurano. Vet Méx 32: 257-263.

12. [INIA] Instituto Nacional de Innovación Agraria. 2009. Conservación y utilización de los recursos genéticos animales: caso cuyes. Programa Nacional de Innovación Agraria en Animales Menores. Estación Experimental Agraria Canaán - Ayacucho. 9 p.

13. Kitagaki M, Yamaguchi M, Nakamura M, Sakurada K, Suwa T, Sasa H. 2005. Age-related changes in haematology and serum chemistry of Weiser-Maples guinea pigs (Cavia porcellus). Lab Anim 39: 321-330. doi: 10.1258/0023677054307042

14. Matute CA. 2019. Determinación de valores de referencia en hemograma y química sanguínea de cuyes machos (Cavia porcellus) en condiciones de altitude. Tesis de Médico Veterinario y Zootecnista. Cuenca, Ecuador: Univ. Politécnica Salesiana. $70 \mathrm{p}$.

15. Medway W, Prier J, Wilkinson J. 1986. Patología clínica veterinaria. México: Ed Hispano-Americana. 532 p.

16. Mendoza R. 2012. Estimación de parámetros productivos y reproductivos de tres ecotipos de cuyes nativos en la Estación Experimental Agraria CANAANINIA. Ayacucho - 2009. Tesis de Médico Veterinario. Ayacucho: Univ. Nacional de San Cristóbal de Huamanga. 77 p.

17. Novozhilov AV, Katyukhin LN, Feizullaev BA. 2012. Dynamics of hematologic parameters and of the erythrocyte deformability index at the juvenal period of rats and guinea pigs. J Evol Biochem Physiol 48: 32-42.

18. Personett AR, Santangelo KS, Kendall LV, Sadar MJ. 2019. Hematologic parameters and blood cultures from the gingival vein compared with the cranial vena cava in Guinea Pigs. J Am Assoc Lab Anim 58: 817-822. doi: 10.30802/ AALAS-JAALAS-19-000039

19. Spittler AP, Afzali MF, Bork SB, Burton LH, Radakovich LB, Seebart $C A$, et al. 2021. Age- and sex-associa- ted differences in hematology and biochemistry parameters of Dunkin Hartley guinea pigs (Cavia porcellus). PLoS One 16: e0253794. doi: 10.1371/ journal.pone.0253794

20. Takisawa M, Chiba J, Haga S, Asano T, Yamamoto N, Honda M. 2004. The normalization of guinea pig leukocyte fractions and lymphocyte subsets in blood and lymphoid tissues using a flow cytometric procedure. Exp Anim 53: 321329. doi: 10.1538/expanim.53.321

21. Tapia JD. 2019. Determinación de valores de referencia en hemograma y química sanguínea de cuyes hembras $(\mathrm{Ca}$ via porcellus) en condiciones de altitud. Tesis de Médico Veterinario y Zootecnista. Cuenca. Ecuador: Univ. Politécnica Salesiana Sede Cuenca. 79 p.

22. Verastegui PA. 2015. Perfil hematológico en cuyes preñadas en la ciudad de Huánuco (Cavia porcellus). Tesis de Médico Veterinario. Huánuco, Perú: Univ. Nacional Hermilio Valdizán. 73 p.

23. Vidalón J. 2014. Evaluación hematológica de dos líneas de selección de cuyes (cárnicos y precoz) criados en la Estación Experimental IVITA el Mantaro. Tesis de Médico Veterinario. Lima, Perú: Univ. Nacional Mayor de San Marcos. 114 p.

24. Washington IM, Hoosier GV. 2012. Clinical biochemistry and hematology. In: The laboratory rabbit, guinea pig, hamster, and other rodents. Seattle, WA, USA: University of Washington. p 57-116.

25. Williams WR, Johnston MS, Higgins S, Izzo1 AA, Kendall LV. 2016. Blood profiles in unanesthetized and anesthetized guinea pigs (Cavia porcellus). Lab Anim (NY) 45: 35-41. doi: 10.1038/laban.911

26. Zimmerman K, Moore DM, Smith SA. 2015. Hematological assessment in pet guinea pigs (Cavia porcellus): blood sample collection and blood cell identification. Vet Clin North Am Exot Anim Pract 18: 33-40. doi: 10.1016/ j.cvex.2014.09.002 